\title{
Fuzzy fault tree reliability analysis based on improved T-S model with application to NC turret
}

\author{
Yue Wu ${ }^{12}$, Zhaojun Yang ${ }^{12}$, Jili Wang ${ }^{12}$, Wei Hu ${ }^{12}$, N. Balakrishnan ${ }^{3}$
}

\begin{abstract}
In Takagi and Sugeno (T-S) fuzzy fault tree analysis (FFTA), the construction of T-S fuzzy gates relies too much on expert experience, which will result in inevitable subjective errors. In order to overcome this disadvantage, a new method was proposed which combined importance index with T-S fuzzy fault tree model to evaluate reliability of the events. The importance index of components can be solved through Monte Carlo (MC) simulation. The proposed method is suitable for systems where exact information on the fault probabilities of the components and the magnitude of failure and effect on system are not available. The concept and calculation method of T-S probability importance was presented. Finally, the feasibility of the method is verified by analyzing the reliability of the sealing subsystem of the NC turret and the weak links of the system are obtained by the importance analysis, which will provide data for system fault diagnosis and preventive maintenance.
\end{abstract}

Keywords Fuzzy fault tree analysis, Takagi and Sugeno model, Monte Carlo simulation, Reliability, NC turret

\section{Introduction}

Fault tree analysis (FTA) is a useful tool for reliability analysis of industrial systems, such as nuclear reactor, aerospace, electric power, chemical and mechanical. A fault tree (FT) is a logic diagram, or directed acyclic graph, that determines the relationship between the top event (i.e., system failure) and the basic events (i.e., component failures). It uses logic gates and events to model how component failures lead to system failures [1-8]. The traditional FTA procedure can be described as 8 steps in Fig.1 [9].

One limitation of the conventional FTA is that the probability of failure of basic events must be known and considered as crisp values. However, in practice, the failure rates of the components are imprecise, deficient or vague in the system modelling [10]. To overcome the limitation in FTA, the fuzzy set theory has been introduced into the FTA (aka. fuzzy fault tree analysis (FFTA)) to deal

$\triangle$ Jili Wang

wangjili@jlu.edu.cn

${ }^{1}$ Key Laboratory of CNC Equipment Reliability, Ministry of Education, Jilin University, Changchun 130025, China

2 School of Mechanical and Aerospace Engineering, Jilin University, Changchun, 130025 China

3 Department of Mathematics and Statistics, McMaster University, Hamilton, Ontario, Canada L8S 4K1 with ambiguous, qualitatively incomplete, ill-defined and inaccurate information.

\begin{tabular}{|c|}
\hline Define the system of interest \\
\hline Define top event of the system \\
Define tree top Structure \\
\hline Explore each branch in successive level of details \\
\hline Solve the fault tree for the combination of events \\
contributing to the top event \\
\hline Identify important dependent failure potentials and \\
adjust the model appropriately \\
\hline Perform quantitative analysis \\
\hline \\
\hline Use the results in decision making \\
\hline
\end{tabular}

Fig.1 Traditional FTA procedure

The fuzzy set theory was introduced in 1965 by Lotfizadeh [11] to deal with uncertainty due to imprecision and vagueness. More and more investigators successfully used the FFTA technique in various areas [12-17]. Lin et al. [12] combined fuzzy set theories with expert elicitation to solve the safety problem of unexpected robot motion in an aircraft wing drilling system. Yazdi et al. [16] established a fuzzy fault tree (FFT) using expert opinion aggregated by fuzzy set theory and applied it to the risk 
assessment of the spherical storage hydrocarbon tank in case of fire and explosion Abad et al. [17] introduced a framework which employed FFTA to identify the causes of a potential change and predict the probability of occurrence of the change in the construction industry.

However, there are still some problems that have not been fully considered:

1. The magnitude of failure and effect on system have not been taken into account. The state of system is only identified in two conditions: either be 0 or 1 (i.e., normal or faulty). However, some failures do not occur randomly, which is dependent on the component degradation process. For this reason, the state of system can be any real value from 0 to 1 .

2. The logic gates are fixed and linguistic information such as the operators' experiences and experts' knowledge cannot be incorporated into the method [18-19].

For these reasons, Song et al. [19] and Yao et al. [20] developed a novel FTA based on the Takagi and Sugeno (T-S) fuzzy model [21]. In their work, fuzzy possibilities are used to describe the events and fuzzy variables are used to describe the magnitude of failure. The T-S fuzzy gates are constructed according to historical data and expert experience to represented the uncertainties in the relationships among events. The use of fuzzy variables and the $\mathrm{T}-\mathrm{S}$ fuzzy gates reduces the need to collect a large number of failure data for computing the failure probabilities. The proposed T-S FFTA method has the advantages in handling imprecision in the probabilities of failure of components arising from inadequate failures data, uncertainties relationships among events and considering the magnitude of failure. Yao et al. [25] introduced T-S gate rules for binary and polymorphic fault trees, extended component importance analysis to T-S FTA, and proposed the concept and calculation of T-S importance. In order to solve the disadvantages of calculation complexity and single inference of T-S FFTA, Chen et al. [26] combined Bayesian network with T-S FTA, proposed a reliability analysis method for multi-state hydraulic system. In addition to calculating the system reliability indices and importance by forward inference, the method can carry out fault diagnosis by backward inference. Bi et al. [27] verified the accuracy of the method combining T-S fuzzy gate fault tree and Bayesian network by comparing with the methods based on traditional Bayesian network and BP neural network. Yao et al. [28] presented an enhanced reliability optimization method based on T-S fault tree and Extended Particle Swarm Optimization (PSO) algorithm that overcomes problems in constructing the reliability optimization model of multi-state systems, as well as early convergence problems in PSO algorithm. Although the above studies can help solve the limitations of FFTA, they also created a new problem that when the historical data is insufficient, the construction of T-S fuzzy gates will inevitably rely too much on expert experience, resulting in inevitable subjective errors.

Therefore, this paper constructs a new T-S fuzzy gates based on the importance index of basic events, which would require as little expert knowledge as possible and reduce the subjective error caused by over-dependence. Finally, the improved T-S FFTA method is constructed according to the above method, and the reliability analysis of the sealing subsystem, which is one of the most important and most faulty components of the numerical control (NC) turret, proves the correctness and provides data for fault diagnosis and preventive maintenance of system.

The remainder of this paper is organized as follows: In Section 2, the T-S fuzzy fault tree model is briefly reviewed. In Section 3, an improved T-S FFTA method is proposed. In Section 4, the T-S probability importance algorithm is described. Then in section 5, a case study about the reliability analysis of NC turret sealing subsystem is didcussed and the effectiveness and superiority of the proposed approach are illustrated. Finally in section 6 , the study is summarized.

\section{T-S Fuzzy Fault Tree}

\subsection{T-S fuzzy fault model}

T-S fuzzy fault tree (FFT) is based on the T-S fuzzy theory. To put it simply, the logic gates such as AND or OR among events are generalized by the T-S fuzzy gates as shown in Fig. 2. 


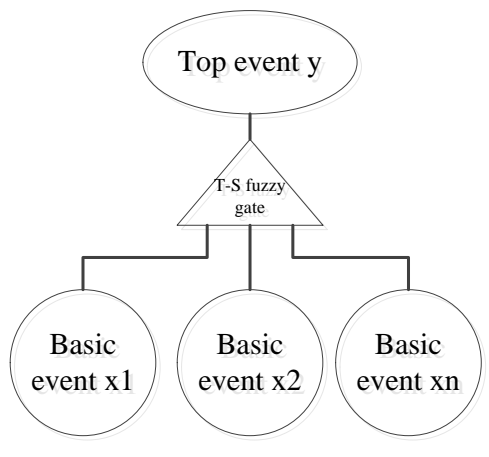

Fig.2 T-S FFT

Let input basic events $x_{1}, x_{2}, \ldots, x_{n}$ and output top event $y$. Then, the fault degree of input and output events are respectively represented by fuzzy numbers $\left(x_{1}^{1}, x_{1}^{2}, \ldots, x_{1}^{k_{1}}\right), \quad \ldots, \quad\left(x_{n}^{1}, x_{n}^{2}, \ldots, x_{n}^{k_{n}}\right) \quad$ and $\left(y^{1}, y^{2}, \ldots, y^{n}\right)$, where $0 \leq x_{1}^{1}<x_{1}^{2}<\ldots<x_{1}^{k_{1}} \leq 1, \ldots$, $0 \leq x_{n}{ }^{1}<x_{n}{ }^{2}<\ldots<x_{n}{ }^{k_{n}} \leq 1, \quad 0 \leq y^{1}<y^{2}<\ldots<y^{n} \leq 1$. Usually, the fault degree can be described by language information as small, medium, large or no fault, degradation, serious fault. Such language information corresponds to the fuzzy numbers $0,0.5$ and 1 respectively.

\subsection{T-S fuzzy gates}

T-S fuzzy theory describes a universal approximation composed of if-then fuzzy rules, which can be represented as follows $[19,29]$ :

$$
\text { If } x \text { is } A \text {, then } y=f(x)
$$

Where $f(x)$ is a linear function of $x, n$ input events are given $m$ fuzzy rules, the rules are shown in Tab. 1:

Table 1 T-S fuzzy gates rules

\begin{tabular}{ccccccccc}
\hline \multirow{2}{*}{ Rules } & $x_{1}$ & $x_{2}$ & $\cdots$ & $x_{n}$ & \multicolumn{5}{c}{$y$} \\
\cline { 6 - 9 } & & & & & $y^{1}$ & $y^{2}$ & $\cdots$ & $y^{k_{y}}$ \\
\hline$l$ & $x_{1}{ }^{a_{1}}$ & $x_{2}{ }^{a_{2}}$ & $\cdots$ & $x_{n}{ }^{{ }_{n}}$ & $P^{l}\left(y^{1}\right)$ & $P^{l}\left(y^{2}\right)$ & $\cdots$ & $P^{l}\left(y^{k_{y}}\right)$ \\
\hline
\end{tabular}

Let the fault status of the input basic event $x_{1}$ be $x_{1}^{a_{1}}, \ldots$, the fault status of the input basic event $x_{n}$ be $x_{n} a_{n}$.Then the occurrence probability of the failure state of output top event $y^{1}$ is $P^{l}\left(y^{1}\right), \ldots$, the occurrence probability of the failure state $y^{k_{y}}$ is $P^{l}\left(y^{k_{y}}\right)$, where $a_{1}=1,2, \ldots, k_{1}, \ldots, \quad a_{n}=1,2, \ldots, k_{n}$.

When the fuzzy possibility of component is known, suppose the fault state of the input basic event is $x_{i}^{a_{i}}(i=1,2, \ldots, n)$ and the occurrence probability of this fault state is $\mathrm{P}\left(x_{\mathrm{i}}=x_{i}^{a_{i}}\right)$, then the execution probability of rule $l$ is:

$$
\mathrm{P}_{0}^{1}=\prod_{i=1}^{n} P\left(x_{i}=x_{i}^{a_{i}}\right)
$$

The occurrence probability of the output top event $y$ corresponding to the fault state is:

$$
\left\{\begin{array}{l}
\mathrm{p}\left(y=y^{1}\right)=\sum_{l=1}^{r} P_{0}^{l} P^{l}\left(y=y^{1}\right) \\
\mathrm{p}\left(y=y^{2}\right)=\sum_{l=1}^{r} P_{0}^{l} P^{l}\left(y=y^{2}\right) \\
\cdots \\
\mathrm{p}\left(y=y^{k_{y}}\right)=\sum_{l=1}^{r} P_{0}^{l} P^{l}\left(y=y^{k_{y}}\right)
\end{array}\right\}
$$

\section{Improved T-S Fuzzy Fault Tree}

To solve the subjective error caused by over-dependence [19-28], this paper constructs a new T-S fuzzy gates based on the importance index of basic events, which would require as little expert knowledge as possible. The importance index of basic events can be solved through Monte Carlo (MC) simulation.

MC simulation is a method that uses random sampling analysis to estimate the problem and to compute the variance of the estimate. By sampling the random variables in the model enough times and analyzing statistical results of the sampling, one can estimate the problem and the variance of the estimated value. This is one of the most effective methods for reliability assessment due to its ability of good expression of statistical nature of the events [30]. The flowchart of the MC simulation algorithm of T-S FFTA model is shown in the Fig.3:

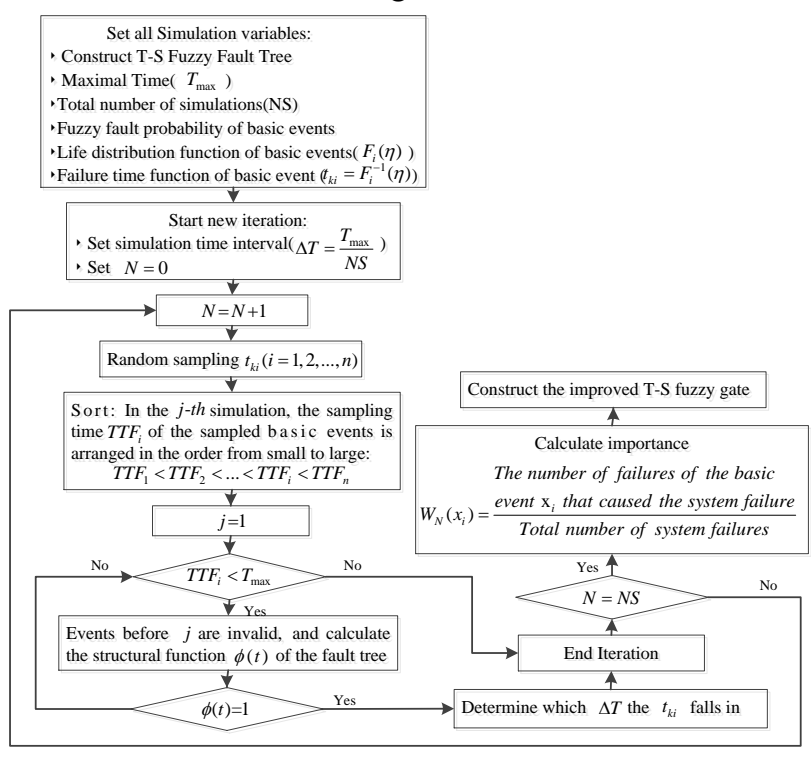

Fig.3 The flowchart of the MC simulation algorithm of T$S$ FFTA model

The construction process of the improved T-S fuzzy gates is as follows:

To simplify the calculation process of T-S fuzzy gate 
rule algorithm, this paper considers the algorithms under three fault states of " 0 ", " 0.5 " and " 1 " of the output event, i.e., $y^{1}=0, y^{2}=0.5, y^{3}=1$.

The greater the importance is, the more easily the components will fail, which is a weak link of the system.

The relationship between the importance interval of the basic events and the influence of the output event in different states can be linearized.

First, we consider the influence of the importance of a single basic event $x_{i}$ to overall output event $y$. When constructing a new T-S fuzzy gates rules, interval judgment is first made on the importance of the input events in the gates rules to obtain the influence value of the corresponding output events in different states, i.e., interval interpolation corresponding to the linear relationship.The results are shown in Tab. 2:

Table 2 Importance influence of a single basic event

\begin{tabular}{|c|c|c|c|c|}
\hline \multirow{2}{*}{$\begin{array}{c}\text { Importance } \\
\text { interval of } \\
x_{i}\end{array}$} & \multirow{2}{*}{$\begin{array}{l}\text { Interval } \\
\text { length }\end{array}$} & \multicolumn{3}{|c|}{$\begin{array}{c}\text { Influence of the output event } y \text { in } \\
\text { different states } O\left(y^{i}\right)\end{array}$} \\
\hline & & $y^{1}=0$ & $y^{2}=0.5$ & $y^{3}=1$ \\
\hline$\left[a_{l}, a_{u}\right)$ & $\Delta l_{m}$ & {$\left[y_{l}^{1}, y_{u}^{1}\right)$} & {$\left[y_{l}^{2}, y_{u}^{2}\right)$} & {$\left[y_{l}^{3}, y_{u}^{3}\right)$} \\
\hline
\end{tabular}

Therefore, we have the fuzzy possibility of the output event in the state of a given rule $l$ in Equation (4):

$$
\left\{\begin{array}{r}
K^{l}\left(y^{i}\right)=\frac{W_{N}\left(x_{i}\right)-a_{i}}{\Delta l_{m}} \cdot \Delta O\left(y^{i}\right)+O\left(y_{l}^{i}\right),\left(y^{i}=0.5,\right. \\
\left.y^{i}=1\right) \\
K^{l}\left(y^{i}\right)=O\left(y_{u}^{i}\right)-\frac{W_{N}\left(x_{i}\right)-a_{i}}{\Delta l_{m}} \bullet \Delta O\left(y^{i}\right), y^{i}=0
\end{array}\right.
$$

Where $K^{l}\left(y^{i}\right)$ represents the fuzzy possibility under the output event $y^{i} ; a_{l}$ represents the lower limit of the interval division of the importance; $a_{u}$ represents the upper limit of the interval division of the importance; $\Delta l_{m}$ is the length of the interval division of the importance; $O\left(y^{i}\right)$ represents the influence of the corresponding state of the output event; $W_{N}\left(x_{i}\right)$ represents the importance of basic event, obtained through MC simulation, whose calculation method is given in Fig.2.

$K^{l}\left(y^{1}\right) 、 K^{l}\left(y^{2}\right) 、 \ldots$ and $K^{l}\left(y^{i}\right)$ satisfy the following relation:

$$
\begin{aligned}
& \frac{K^{l}\left(y^{1}\right)}{K^{l}\left(y^{1}\right)+K^{l}\left(y^{2}\right)+\ldots+K^{l}\left(y^{i}\right)}+\ldots \\
& +\frac{K^{l}\left(y^{i}\right)}{K^{l}\left(y^{1}\right)+K^{l}\left(y^{2}\right)+\ldots+K^{l}\left(y^{i}\right)}=1
\end{aligned}
$$

Then we consider the influence of correlation among components in a single gate rule:
When two or more components are in the fault state of $y^{i}$ at the same time, the calculation of the fuzzy possibility of the output events shall be judged by adding the importance values of all components in the fault state of $y^{i}$. The calculation process is the same as the case of a single component.

\section{$4 \mathrm{~T}-\mathrm{S}$ probability importance analysis}

Importance is an important part of FTA quantitative analysis. It describes the contribution of a component failure to the occurrence probability of top events, which is based upon time, component reliability parameters, and the system structure. Importance can not only be used for reliability analysis of the system, but also for optimization design of the system and guidance for maintenance and diagnosis of the system.

An algorithm for determining the probability importance of T-S FFTA is studied. When the fuzzy probability of basic event $x_{j}{ }^{\prime} s$ fault state $x_{j}^{i_{j}}$ is $P\left(x_{j}^{i_{j}}\right)\left(i_{j}=1,2, \ldots, k_{j}\right)$, the T-S probability importance $I_{T_{q}}^{\mathrm{Pr}}\left(x_{j}{ }^{\mathrm{i}_{j}}\right)$ in fault state $T_{q}$ of fault tree top event $T$ is: $I_{T_{q}}^{\mathrm{Pr}}\left(x_{j}{ }^{\mathrm{i}_{j}}\right)=P\left(T_{q}, P\left(x_{j}{ }^{\mathrm{i}_{j}}\right)=1\right)-P\left(T_{q}, P\left(\mathrm{x}_{\mathrm{j}}{ }^{\mathrm{i}_{j}}\right)=0\right)$

Where $P\left(T_{q}, P\left(x_{j}{ }^{\mathrm{i}_{j}}\right)=1\right) \quad$ represents the fuzzy possibility at the fault state $T_{q}$ of the top event $T$ when the component $x_{j}$ fault state is $P\left(x_{j}^{i_{j}}\right)=1$; $P\left(T_{q}, P\left(x_{j}^{i_{j}}\right)=0\right)$ represents the fuzzy possibility of at the fault state $T_{q}$ of the top event $T$ when the component $x_{j}$ fault state is $P\left(x_{j}^{i_{j}}\right)=0$. It can be understood that the fuzzy possibility $T_{q}$ at the fault state is $T_{q}$ irrelevant with $x_{j} \cdot I_{T_{q}}^{\mathrm{Pr}}\left(x_{j}{ }^{i_{j}}\right)$ can also be considered as the fuzzy possibility that component $x_{j}$ alone causes top event $T$ with fault state $x_{j}^{i_{j}}$ when the fault state is $T_{q}$.

By calculating the T-S probability importance of basic event $x_{j}$ in each fault state, the T-S probability importance $I_{T_{q}}^{\mathrm{Pr}}\left(x_{j}\right)$ of top event $T$ is obtained as

$$
I_{T_{q}}^{\mathrm{Pr}}\left(x_{j}\right)=\frac{\sum_{i_{j}=1}^{k_{j}{ }^{\prime}} I_{T_{q}}^{\operatorname{Pr}}\left(x_{j}^{i_{j}}\right)}{k_{j}{ }^{\prime}}
$$

Where $k_{j}{ }^{\prime}$ is the number of fault states in which the $j$ - 
th event is not 0 .

\section{Case study}

As a core functional component of $\mathrm{CNC}$ lathe, the $\mathrm{NC}$ turret is one of the most prone to failure, whether they can operate safely affects the function and efficiency of the CNC lathe directly. NC turret can be divided into five function modules according to its composition and working principle. The sealing system is the fault-prone component. Therefore, the reliability analysis of NC turret sealing subsystem is discussed using the improved T-S FFTA method in this paper.

\subsection{T-S FFTA model for NC turret sealing subsystem}

The T-S fuzzy fault tree was constructed with the failure of the sealing subsystem as the top event, as shown in Fig.4.

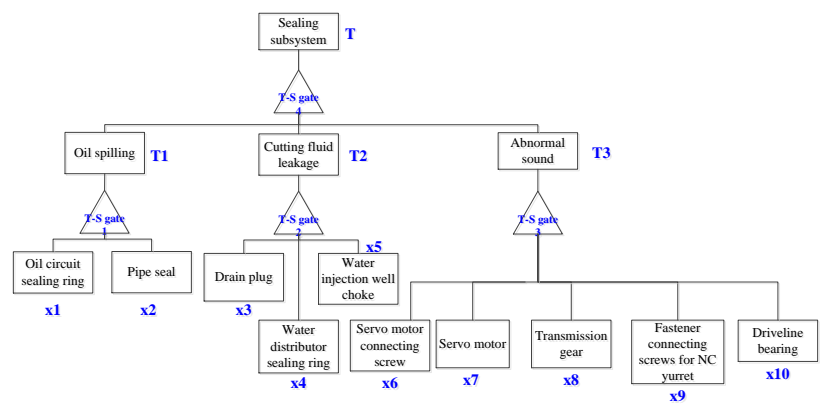

Fig. 4 T-S FFT of NC turret sealing subsystem

The reliability data for the components of the sealing subsystem are shown in Tab.3[31].

Table 3 Reliability data for the components of sealing subsystem

\begin{tabular}{clcc}
\hline Components & $\begin{array}{c}\text { Fuzzy failure } \\
\text { probability }\end{array}$ & Components & $\begin{array}{c}\text { Fuzzy failure } \\
\text { probability }\end{array}$ \\
\hline X1 & $3.898 \mathrm{E}-05$ & $\mathrm{X} 6$ & $1.731 \mathrm{E}-07$ \\
$\mathrm{X} 2$ & $1.27 \mathrm{E}-05$ & $\mathrm{X} 7$ & $1.27 \mathrm{E}-06$ \\
$\mathrm{X} 3$ & $6.923 \mathrm{E}-06$ & $\mathrm{X} 8$ & $1.032 \mathrm{E}-06$ \\
X4 & $1.129 \mathrm{E}-05$ & $\mathrm{X} 9$ & $1.169 \mathrm{E}-05$ \\
X5 & $1.74 \mathrm{E}-06$ & $\mathrm{X} 10$ & $1.322 \mathrm{E}-06$ \\
\hline
\end{tabular}

\subsection{MC reliability simulation of NC turret sealing} subsystem

Through the trial algorithm, determining input parameters: $\mathrm{NS}=100000$ times and the maximum working time $\mathrm{T}_{\max }=150000 \mathrm{~h}$.

According to the MC simulation process in Fig.3, the life distribution function of the 10 basic events of the $\mathrm{NC}$ turret sealing subsystem was randomly sampled and sorted and the simulation curve of the importance of the basic events was shown in Fig.5. The stable value of the importance of the basic events was shown in Tab.4.

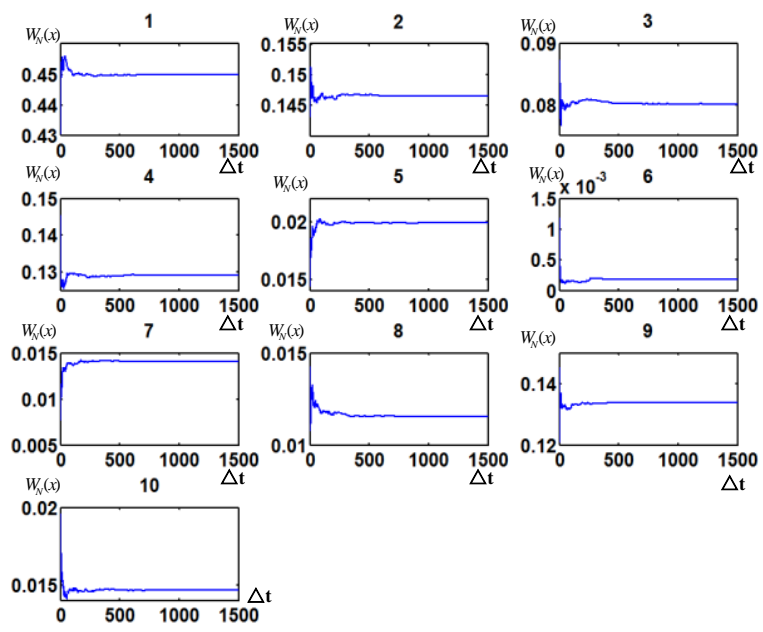

Fig. 5 Importance distribution curve of basic events

Table 4 Importance for the components of sealing subsystem

\begin{tabular}{cccccc}
\hline Component & $\mathrm{X} 1$ & $\mathrm{X} 2$ & $\mathrm{X} 3$ & $\mathrm{X} 4$ & $\mathrm{X} 5$ \\
\hline Importance & 0.4477 & 0.1450 & 0.12864 & 0.02018 & 0.08001 \\
\hline Component & $\mathrm{X} 6$ & $\mathrm{X} 7$ & $\mathrm{X} 8$ & $\mathrm{X} 9$ & $\mathrm{X} 10$ \\
\hline Importance & 0.00029 & 0.01476 & 0.01185 & 0.0435 & 0.13596 \\
\hline
\end{tabular}

5.3 Reliability analysis of improved T-S FFTA for NC turret sealing subsystem

The criteria for dividing the importance interval can be obtained by Tab.2 and Tab.4, as shown in Tab.5.

Table 5 Criteria for dividing the importance interval

\begin{tabular}{|c|c|c|c|c|}
\hline \multirow{2}{*}{$\begin{array}{c}\text { Pattern } \\
\text { importance } \\
\text { interval } \\
{\left[a_{l}, a_{u}\right)}\end{array}$} & \multirow{2}{*}{$\begin{array}{c}\text { Interval } \\
\text { length } \\
\qquad l_{m}\end{array}$} & \multicolumn{3}{|c|}{ Influence of the output event $y$ in } \\
\hline & & $y^{1}=0$ & $y^{2}=0.5$ & $y^{3}=1$ \\
\hline$[0,0.01)$ & 0.01 & {$[0.85,1)$} & {$[0,0.1)$} & {$[0,0.05)$} \\
\hline$[0.01,0.05)$ & 0.04 & {$[0.75,0.85)$} & {$[0.1,0.15)$} & {$[0.05,0.1)$} \\
\hline$[0.05,0.1)$ & 0.05 & {$[0.65,0.75)$} & {$[0.15,0.2)$} & {$[0.1,0.15)$} \\
\hline$[0.1,0.25)$ & 0.15 & {$[0.45,0.65)$} & {$[0.2,0.35)$} & {$[0.15,0.3)$} \\
\hline$[0.25,0.5)$ & 0.25 & {$[0.28,0.45)$} & {$[0.35,0.55)$} & {$[0.3,0.48)$} \\
\hline$[0.5,0.7)$ & 0.2 & {$[0.12,0.28)$} & {$[0.55,0.72)$} & {$[0.48,0.62)$} \\
\hline$[0.7,0.9)$ & 0.2 & {$[0,0.12)$} & {$[0.72,0.88)$} & {$[0.62,0.8)$} \\
\hline
\end{tabular}

The calculation process for T-S gate 1 is provided below. According to Tab.4, it is known that the importance of basic events $x_{1}$ and $x_{2}$ obtained from MC simulation are respectively $W_{N}\left(x_{1}\right)=0.4477, W_{N}\left(x_{2}\right)=0.1450$, then it is determined from Tab.5 that the importance of basic events $x_{1}$ and $x_{2}$ fall in the interval [0.25,0.5)、 
$[0.1,0.25)$. It can be calculated that when the fauil states of basic events are $x_{1}=0.5, x_{2}=0$, the fuzzy possibility of the corresponding state $y^{1}=0 、 y^{2}=0.5$ and $y^{3}=1$ of the output event $T_{1}$ is:

$$
\left\{\begin{array}{l}
K^{l}\left(y^{1}=0\right)=0.45-\frac{0.4477-0.25}{0.25} \times 0.17=0.32 \\
K^{l}\left(y^{2}=0.5\right)=\frac{0.4477-0.25}{0.25} \times 0.2+0.35=0.51 \\
K^{l}\left(y^{3}=1\right)=\frac{0.4477-0.25}{0.25} \times 0.18+0.3=0.44
\end{array}\right.
$$

The results are normalized as follows:

$$
\left\{\begin{array}{l}
K^{l}\left(y^{1}=0 \mid x_{1}=0.5, \quad x_{2}=0\right)=0.25 \\
K^{l}\left(y^{2}=0.5 \mid x_{1}=0.5, \quad x_{2}=0\right)=0.40 \\
K^{l}\left(y^{3}=1 \mid x_{1}=0.5, \quad x_{2}=0\right)=0.35
\end{array}\right.
$$

Similarly, when the fauil states of basic events are $x_{1}=0, x_{2}=0.5$, the fuzzy possibility corresponding to the output event $T_{1}$ in different states can be calculated as follows:

$$
\left\{\begin{array}{l}
K^{l}\left(y^{1}=0 \mid x_{1}=0, \quad x_{2}=0.5\right)=0.57 \\
K^{l}\left(y^{2}=0.5 \mid x_{1}=0, \quad x_{2}=0.5\right)=0.24 \\
K^{l}\left(y^{3}=1 \mid x_{1}=0, \quad x_{2}=0.5\right)=0.19
\end{array}\right.
$$

When the fauil states of basic events are $x_{1}=0.5, x_{2}=0.5$, the sum of importance of $x_{1}$ and $x_{2}$ is:

$$
\sum W_{N}(\mathrm{X})=W_{N}\left(x_{1}\right)+W_{N}\left(x_{2}\right)=0.5927
$$

Then the fuzzy possibility corresponding to the output event $T_{1}$ in different states can be calculated as follows:

$$
\left\{\begin{array}{l}
K^{l}\left(y^{1}=0 \mid x_{1}=0.5, \quad x_{2}=0.5\right)=0.15 \\
K^{l}\left(y^{2}=0.5 \mid x_{1}=0.5, \quad x_{2}=0.5\right)=0.46 \\
K^{l}\left(y^{3}=1 \mid x_{1}=0.5, \quad x_{2}=0.5\right)=0.39
\end{array}\right.
$$

According to the above analysis, the new T-S fuzzy gate1 can be shown in Tab.6. The new T-S fuzzy gate2-4 can be shown as Tab.7 - Tab.9 in a similar way.

Table 6 T-S gate rules 1 for NC turret sealing subsystem

\begin{tabular}{cccccc}
\hline \multirow{2}{*}{ Rule } & $\mathrm{X} 1$ & $\mathrm{X} 2$ & \multicolumn{4}{c}{$\mathrm{T} 1$} \\
\cline { 4 - 6 } & & & 0 & 0.5 & 1 \\
\hline 1 & 0 & 0 & 1 & 0 & 0 \\
2 & 0 & 0.5 & 0.572765577 & 0.2378886 & 0.1893458 \\
3 & 0 & 1 & 0 & 0 & 1 \\
4 & 0.5 & 0 & 0.249261325 & 0.4013613 & 0.3493773 \\
& & & $\ldots$ & & \\
9 & 1 & 1 & 0 & 0 & 1 \\
\hline
\end{tabular}

Table 7 T-S gate rules 2 for NC turret sealing subsystem

\begin{tabular}{ccccccc}
\hline \multirow{2}{*}{ Rule } & $\mathrm{X} 3$ & $\mathrm{X} 4$ & $\mathrm{X} 5$ & \multicolumn{4}{c}{$\mathrm{T} 2$} \\
\cline { 5 - 7 } & & & & 0 & 0.5 & 1 \\
\hline 1 & 0 & 0 & 0 & 1 & 0 & 0 \\
2 & 0 & 0 & 0.5 & 0.82455 & 0.112725 & 0.062725 \\
3 & 0 & 0 & 1 & 0 & 0 & 1 \\
4 & 0 & 0.5 & 0 & 0.6003506 & 0.2243563 & 0.1752931 \\
5 & 0 & 0.5 & 0.5 & 0.56647 & 0.240977 & 0.192553 \\
27 & 1 & 1 & 1 & 0 & 0 & 1 \\
\hline
\end{tabular}

Table 8 T-S gate rules 3 for NC turret sealing subsystem

\begin{tabular}{ccccccccc}
\hline \multirow{2}{*}{ Rule } & $\mathrm{X} 6$ & $\mathrm{X} 7$ & $\mathrm{X} 8$ & $\mathrm{X} 9$ & $\mathrm{X} 1$ & \multicolumn{4}{c}{$\mathrm{T} 3$} \\
\cline { 6 - 8 } & & & & & 0 & 0 & 0.5 & 1 \\
\hline 1 & 0 & 0 & 0 & 0 & 0 & 1 & 0 & 0 \\
2 & 0 & 0 & 0 & 0 & 0.5 & 0.5879 & 0.2304 & 0.1816 \\
3 & 0 & 0 & 0 & 0 & 1 & 0 & 0 & 1 \\
4 & 0 & 0 & 0 & 0.5 & 0 & 0.7662 & 0.1418 & 0.0918 \\
5 & 0 & 0 & 0 & 0.5 & 0.5 & 0.5166 & 0.2654 & 0.2179 \\
6 & 0 & 0 & 0 & 0.5 & 1 & 0 & 0 & 1 \\
7 & 0 & 0 & 0 & 1 & 0 & 0 & 0 & 1 \\
8 & 0 & 0 & 0 & 1 & 0.5 & 0 & 0 & 1 \\
243 & 1 & 1 & 1 & 1 & 1 & 0 & 0 & 1 \\
\hline
\end{tabular}

Table 9 T-S gate rules 4 for NC turret sealing subsystem

\begin{tabular}{ccccccc}
\hline & & & \multicolumn{5}{c}{$\mathrm{T}$} \\
\cline { 5 - 7 } 序号 & T1 & T2 & T3 & 0 & 0.5 & 1 \\
\hline 1 & 0 & 0 & 0 & 1 & 0 & 0 \\
2 & 0 & 0 & 0.5 & 0.4745387 & 0.2860754 & 0.2393859 \\
3 & 0 & 0 & 1 & 0 & 0 & 1 \\
4 & 0 & 0.5 & 0 & 0.440402 & 0.3028217 & 0.2567763 \\
5 & 0 & 0.5 & 0.5 & 0.2581087 & 0.396757 & 0.3451344 \\
& & & & $\ldots$ & & 1 \\
\hline
\end{tabular}

According to Eq.(3), the fuzzy probability of top event at different fault degrees can be calculated, as shown in Table 10. The result illustrates that the failure possibilities of the sealing subsystem have the same magnitude as that of its components, and the possibility of system failure with a magnitude of 1 is greater than that for the oil spilling, cutting fluid leakage and abnormal sound, which are similar to that obtained from the actual system. 
Table 10 The fuzzy possibility at different failure degrees of the top events

\begin{tabular}{cccccccccc}
\hline $\begin{array}{c}\text { Fuzzy } \\
\text { possibility } \\
\left(10^{-6}\right)\end{array}$ & \multicolumn{2}{c}{$\mathrm{T} 1$} & \multicolumn{2}{c}{$\mathrm{T} 2$} & \multicolumn{2}{c}{$\mathrm{T} 3$} & \multicolumn{2}{c}{$\mathrm{T}$} \\
\cline { 2 - 10 } & 0.5 & 1 & 0.5 & 1 & 0.5 & 1 & 0.5 & 1 \\
\hline$P$ & 18.7 & 67.7 & 3.98 & 22.9 & 2.2 & 16.9 & 10.3 & 116 \\
\hline
\end{tabular}

Combined with Eq.(6), the T-S probability importance of NC turret sealing subsystem is calculated. The results are shown in Table 11:

Table 11 T-S probability importance of each component in different fault states

\begin{tabular}{cccccc}
\hline $\begin{array}{c}\text { Probability } \\
\text { importance }\end{array}$ & $\mathrm{X} 1$ & $\mathrm{X} 2$ & $\mathrm{X} 3$ & $\mathrm{X} 4$ & $\mathrm{X} 5$ \\
\hline$I_{0.5}^{\mathrm{Pr}}\left(x_{j}\right)$ & 0.09147 & 0.05422 & 0.02725 & 0.03397 & 0.01706 \\
$I_{1}^{\mathrm{Pr}}\left(x_{j}\right)$ & 0.75400 & 0.64171 & 0.58816 & 0.61649 & 0.54592 \\
\hline $\begin{array}{c}\text { Probability } \\
\text { importance }\end{array}$ & $\mathrm{X} 6$ & $\mathrm{X} 7$ & $\mathrm{X} 8$ & $\mathrm{X} 9$ & $\mathrm{X} 10$ \\
\hline$I_{0.5}^{\mathrm{Pr}}\left(x_{j}\right)$ & 0.00041 & 0.01515 & 0.01463 & 0.02029 & 0.03296 \\
$I_{1}^{\mathrm{Pr}}\left(x_{j}\right)$ & 0.50110 & 0.54069 & 0.53843 & 0.56295 & 0.61843 \\
\hline
\end{tabular}

As can be seen from Tab.11, when the failure states of NC turret sealing subsystem are 0.5 and 1 respectively, the order of probability importance of each component from large to small is as follows:

When failure status is $0.5, \mathrm{X} 1>\mathrm{X} 2>\mathrm{X} 4>\mathrm{X} 10>\mathrm{X} 3>\mathrm{X} 9>\mathrm{X} 5$ $>$ X7>X8>X6;

When failure status is $1, \mathrm{X} 1>\mathrm{X} 2>\mathrm{X} 10>\mathrm{X} 4>\mathrm{X} 3>\mathrm{X} 9>\mathrm{X} 5$ $>\mathrm{X} 7>\mathrm{X} 8>\mathrm{X} 6$

\subsection{Result Comparison}

Statistical analysis was made on 152 post-sale data of SLT $\mathrm{NC}$ turret in a CNC machine tool factory, among which the fault frequency of NC turret sealing subsystem was the highest, accounting for $35 \%$ of the total fault data. The failure frequency distribution of its components is shown in Fig. 6[32].

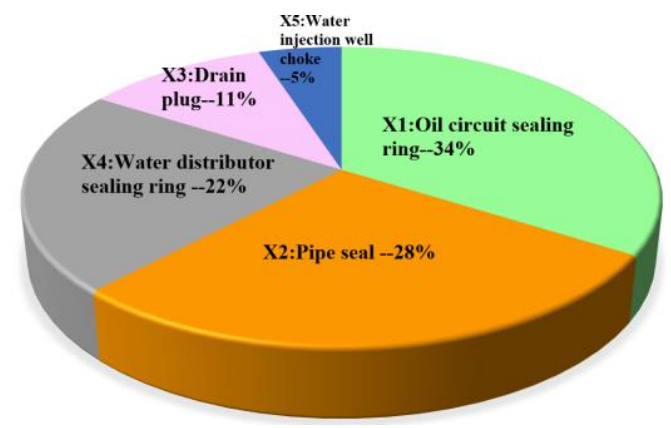

Fig. 6 Failure frequency distribution of sealing subsystem components

It can be found from the actual fault data that the failure frequency of the key components of the NC turret sealing subsystem is sorted as follows: $\mathrm{X} 1>\mathrm{X} 2>\mathrm{X} 4>\mathrm{X} 3>\mathrm{X} 5$.

The result is consistent with the ranking of the system in the "1" fault state obtained by the improved T-S FFTA method, which verifies the correctness of the method.

The greater the importance is, the more easily the components will fail, which is a weak link of the system. It can be seen that when the system is in the " 0.5 " fault state, the oil circuit sealing ring has the greatest impact on the system, followed by the pipe seal, water distributor sealing ring and driveline bearing, so when the system runs to the set preventive maintenance time point, the system needs to be incompletely repaired, and the abovementioned parts should be repaired and replaced first to extend the service cycle of the system.

When the system is in the "1" fault state, the oil circuit sealing ring has the greatest impact on the system, followed by pipe seal, driveline bearing and water distributor sealing ring, servo motor connecting screws have the least impact.

\section{Conclusions}

An improved T-S FFTA had been provided in this paper by introducing importance index of basic events with T-S FFTA. The proposed method, in which fuzzy possibilities are used to describe the events, fuzzy variables are used to describe the magnitude of failure and the T-S fuzzy gates are constructed to represented the uncertainties in the relationships among events, could not only solve the problem of imprecision in the probabilities of failure of components arising from inadequate failures data, uncertainties relationships among events and considering the magnitude of failure, but also require as little expert knowledge as possible and reduce the subjective error caused by over-dependence. T-S probability importance analysis of the basic events at different fault magnitude indicates that oil circuit sealing ring, pipe seal, driveline 
bearing and water distributor sealing ring play important roles in affecting failure of the NC turret sealing subsystem.

\section{Acknowledgements}

This work was supported by National Science and Technology Major Project (Grant No. 2019ZX04005001009) and China Scholarship Council (Grant No. 202006170069).

\section{Conflicts of interest}

Not applicable

\section{Availability of data and material}

Not applicable

\section{Code availability}

Not applicable

\section{Authors' contributions}

Yue Wu: Background research, Data curation, Software, Validation writing-original draft, Editing.

Zhaojun Yang: Methodology, Review \& editing, Supervision.

Jili Wang: Supervision, Project administration, Funding acquisition

Wei Hu: Assist in experiment, Data curation, Review \& editing

N. Balakrishnan: Supervision, Review \& editing

All authors read and approved the final manuscript.

\section{Ethics approval}

Not applicable

\section{Consent to participate}

Not applicable

\section{Consent for publication}

Not applicable

\section{Reference}

[1] W. S. Lee, D. L. Grosh, F. A. Tillman and C. H. Lie, "Fault Tree Analysis, Methods, and App lications-A Review," IEEE Transactions on Reli ability, vol. R-34, no. 3, pp. 194-203, Aug. 198
5, doi: 10.1109/TR.1985.5222114.

[2] Mahmood, Y. A. et al. "Fuzzy fault tree analysi s: a review of concept and application." Internat ional Journal of System Assurance Engineering \& Management 4.1(2013):19-32.

[3] Vaurio, J. K. "Common cause failure probabilitie $\mathrm{s}$ in standby safety system fault tree analysis wi th testing — scheme and timing dependencies." Re liability Engineering \& System Safety 79.1(2003): 43-57.

[4] Gupta, Suprakash, and Jayanta Bhattacharya. "Re liability analysis of a conveyor system using hy brid data." Quality and Reliability Engineering I nternational 23.7 (2007): 867-882.

[5] Huang, Hong-Zhong, Xin Tong, and Ming J. Zu o. "Posbist fault tree analysis of coherent system s." Reliability Engineering \& System Safety 84.2 (2004): 141-148.

[6] Chang, J-R., et al. "The reliability of general va gue fault-tree analysis on weapon systems fault diagnosis." Soft Computing 10.7 (2006): 531-542.

[7] Jenab, Kouroush, and Balbir S. Dhillon. "Stocha stic fault tree analysis with self-loop basic event s." IEEE Transactions on Reliability 54.1 (2005): 173-180.

[8] Ruijters, Enno, and Mariëlle Stoelinga. "Fault tre e analysis: A survey of the state-of-the-art in $\mathrm{m}$ odeling, analysis and tools." Computer science $r$ eview 15 (2015): 29-62.

[9] Ayyub, Bilal M. Risk analysis in engineering an d economics. Crc Press, 2014.

[10] Mentes, Ayhan, and Ismail H. Helvacioglu. "An application of fuzzy fault tree analysis for sprea d mooring systems." Ocean Engineering 38.2-3 (2011): 285-294.

[11] Zadeh, Lotfi Asker. "Fuzzy sets as a basis for a theory of possibility." Fuzzy sets and systems 1.1 (1978): 3-28.

[12] Lin, Ching-Torng, and Mao-Jiun J. Wang. "Hybr id fault tree analysis using fuzzy sets." Reliabilit y Engineering \& System Safety 58.3 (1997): 20 5-213.

[13] Hosseini, Navid, Saeed Givehchi, and Reza Mak noon. "Cost-based fire risk assessment in natural 
gas industry by means of fuzzy FTA and ETA.

" Journal of Loss Prevention in the Process Ind ustries 63 (2020): 104025.

[14] Kabir, Sohag, et al. "Uncertainty-aware dynamic reliability analysis framework for complex syste ms." IEEE Access 6 (2018): 29499-29515.

[15] Nadjafi, Mohammad, Mohammad Ali Farsi, and Hossein Jabbari. "Reliability analysis of multi-sta te emergency detection system using simulation approach based on fuzzy failure rate." Internatio nal Journal of System Assurance Engineering an d Management 8.3 (2017): 532-541.

[16] Yazdi, Mohammad, Orhan Korhan, and Sahand Daneshvar. "Application of fuzzy fault tree analy sis based on modified fuzzy AHP and fuzzy TO PSIS for fire and explosion in the process indus try." International journal of occupational safety and ergonomics 26.2 (2020): 319-335.

[17] Abad, Fatemeh, Ehsan Eshtehardian, and Katayo un Taghizade. "Framework for proactive change management: Assessing the risk of change in co nstruction projects using fuzzy fault tree analysis. " Journal of Architectural Engineering 25.2 (201 9): 04019010.

[18] Pan, HanSuk, and WonYoung Yun. "Fault tree a nalysis with fuzzy gates." Computers \& industria 1 engineering 33.3-4 (1997): 569-572.

[19] Song, Hua, Hong-Yue Zhang, and C. W. Chan. "Fuzzy fault tree analysis based on $\mathrm{T}-\mathrm{S}$ model with application to INS/GPS navigation system." Soft computing 13.1 (2009): 31-40.

[20] Yao, Chengyu, and Yingyi Zhang. "TS Model B ased Fault Tree Analysis on the Hoisting Syste m of Rubber-Tyred Girder Hoister." 2010 WASE International Conference on Information Engine ering. Vol. 4. IEEE, 2010.

[21] Takagi, Tomohiro, and Michio Sugeno. "Fuzzy i dentification of systems and its applications to modeling and control." IEEE transactions on sys tems, man, and cybernetics 1 (1985): 116-132.

[22] Bououden, Sofiane, Mohammed Chadli, and $\mathrm{Ha}$ mid Reza Karimi. "Control of uncertain highly nonlinear biological process based on Takagi-Su geno fuzzy models." Signal Processing 108 (201 5): $195-205$.

[23] Mollov, Stanimir, et al. "Effective optimization $f$ or fuzzy model predictive control." IEEE Transa ctions on fuzzy systems 12.5 (2004): 661-675.

[24] Wang, Jiahui, et al. "Sliding mode control for $\mathrm{n}$ on-linear systems by Takagi-Sugeno fuzzy mode 1 and delta operator approaches." IET Control T heory \& Applications 11.8 (2017): 1205-1213.

[25] TAO, Cheng. "Importance Analysis Method of F uzzy Fault Tree Based on TS Model." China M echanical Engineering 22.11 (2011): 1261.

[26] CHEN, Dong. "Reliability analysis of multi-state hydraulic system based on TS fuzzy fault tree a nd Bayesian network." China Mechanical Engine ering 24.07 (2013): 899.

[27] $\mathrm{Bi}$, Zhuqing, et al. "Research on fault diagnosis for pumping station based on TS fuzzy fault tre e and bayesian network." Journal of Electrical a nd Computer Engineering 2017 (2017).

[28] Yao, Chengyu, Bin Wang, and Dongning Chen. "Reliability optimization of multi-state hydraulic system based on TS fault tree and extended PS O algorithm." IFAC Proceedings Volumes 46.5 (2013): 463-468.

[29] Wang, Li-Xin. Adaptive fuzzy systems and contr ol: design and stability analysis. Prentice-Hall, I nc., 1994.

[30] Tabesh, Massoud, et al. "Risk Assessment of W ater Treatment Plants Using Fuzzy Fault Tree A nalysis and Monte Carlo Simulation." Iranian Jo urnal of Science and Technology, Transactions o f Civil Engineering (2021): 1-16.

[31] Hu, Wei, et al. "A New and Practical Reliability Allocation Method for a Complex System of $\mathrm{N}$ C Turrets." Mathematical Problems in Engineerin g 2019 (2019).

[32] Yue Wu. "Reliability Analysis of CNC Turret B ased on MC and T-S Fusion Polymorphic Fault Tree." Jilin University 2019. 